\title{
Evaluation of contact lenses for central vision in high myopia
}

\author{
GERALD FONDA
}

New Fersey, U.S.A.

Contact lenses do not significantly improve central vision as compared to spectacle lenses. I have never rehabilitated a highly myopic patient with subnormal vision by prescribing contact lenses as an optical aid, nor have I ever seen a patient so rehabilitated. My clinical experience confirms theoretical calculations that the effective magnification is not greater than 27 per cent. for a spectacle correction of $-20 \mathrm{D}$. This study presents data supporting this statement based upon my own clinical tests.

I suspect that what are believed to be dramatic improvements made by contact lenses can in many cases be attributed to an incorrect spectacle correction or to inaccurate vision testing. In 1958, I was enthusiastic about the use of contact lenses for improving the vision of patients with subnormal vision associated with high myopia, because at that time I believed that there was a 25 per cent. increase in magnification for every ro D of myopia with the spectacle correction. I became disenchanted by my clinical experience since I could never obtain this degree of improvement. Later, a better understanding of the theory convinced me that I should not expect so great a degree of magnification. The graph published by Boeder (1938) showing a magnification of 46 per cent. for a $-20 \mathrm{D}$ myope has been misinterpreted to refer to the spectacle correction. At a vertex distance of $12 \mathrm{~mm}$. from the cornea the spectacle correction corresponding to $-20 \mathrm{D}$ on the cornea is $-26 \cdot 25 \mathrm{D}$, and it is to this that the 46 per cent. magnification refers. Boeder's figures do not represent a comparison between contact lenses and spectacle lenses but give the retinal image size in the myope corrected with a contact lens as compared with that of the standard emmetropic eye.

\section{Theory of lens correction of ametropia and magnification}

A lens must form an image of the distant object at the far point of the eye in order to correct the ametropia. For any given eye the size of the retinal image is directly proportional to the size of the image formed at the far point of the eye. This discussion will be limited to the correction of myopia because it is our specific interest.

Fig. I shows that the second principal focus $\left(F_{2}\right)$ of the lens must coincide with the far point (FP) of the eye in order to form the image of the distant object at the far point. The image at the far point determines the size of the retinal image (RI).

The power of the lens which is needed to make the second principal focus coincide with the far point of the eye will depend upon the degree of myopia of the eye and the distance between the lens and the cornea. Fig. 2 represents the effect of the difference in distance of the correcting lenses from the cornea, i.e. the vertex distance, which produces the magnification.

Received for publication December 5, 1972

Address for reprints: G. Fonda, M.D., 551 Millburn Avenue, Short Hills, New Jersey 07078, U.S.A.

Paper presented at a meeting of the Contact Lens Association of Ophthalmology at Las Vegas, Nevada, on September 19, 1971 
RI - Retinal image

FP-Far point of eye

$F_{2}$ - Secondary principal focus of lens

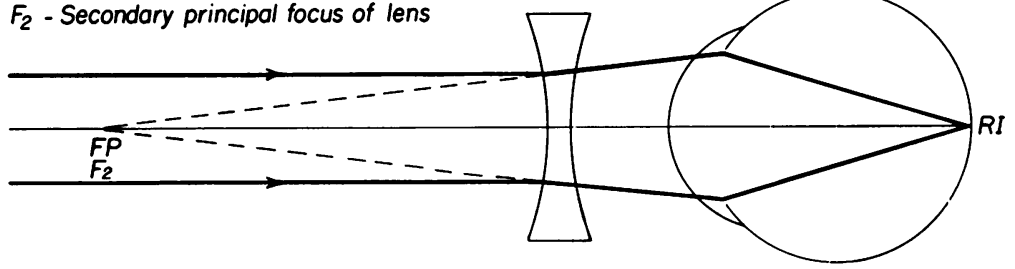

FIG. I Lens correction of myopia

$f_{2(e)}$ - Focal distance of contact lens

fe(so) - Focal distonce of spectacle lens

$I_{c l}$-Image of contact lens

Isp-Image of spectacle lens

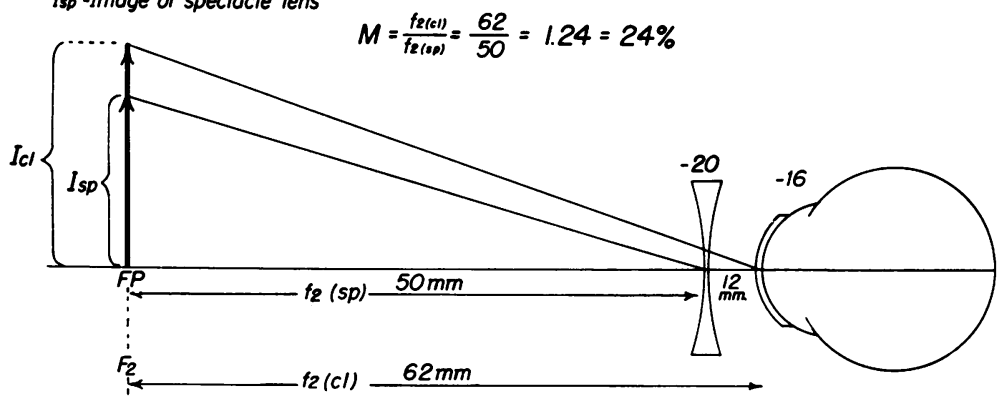

FIG. 2 Magnification of contact lens in comparison with spectacle lens

Fig. 2 illustrates magnification at the far point (FP) of the eye produced by comparing the size of the image formed by the contact lens (Icl) to the image formed by the spectacle lens (Isp). Of the two images, the larger is formed by the weaker contact lens (Icl) with the longer focal length and the smaller by the stronger spectacle lens (Isp) with the shorter focal length. From this we may conclude that the size of the retinal image is directly proportional to the focal lengths and indirectly to the power of the correcting lenses in dioptres.

Since the method of calculating the degree of magnification is based upon this illustration, an example is presented.

GIVEN

Spectacle correction $-20 \mathrm{D}$

Vertex distance $\quad 12 \mathrm{~mm}$. SOLVE FOR:

(1) Focal length of $-20 \mathrm{D}$ spectacle lens $=\frac{1,000}{20}=50 \mathrm{~mm}$.

$$
\begin{aligned}
\text { (2) Magnification } & =\frac{\mathrm{f} 2(\mathrm{cl})}{\mathrm{f} 2(\mathrm{sp})}=\frac{62}{50}=1 \cdot 24 \text { per cent. } \\
\text { (3) Power of contact lens } & =\frac{1,000}{62}=16 \cdot 13 \mathrm{D}
\end{aligned}
$$

Fig. 3 (opposite) summarizes the magnifications calculated by the above method for spectacle corrections ranging from -40 to $+20 \mathrm{D}$ at a vertex distance of $12 \mathrm{~mm}$.

Fig. 3 shows that the retinal image is increased (magnified) in myopia but decreased (diminished) in hyperopia. Note that the magnification for contact lenses in comparison 


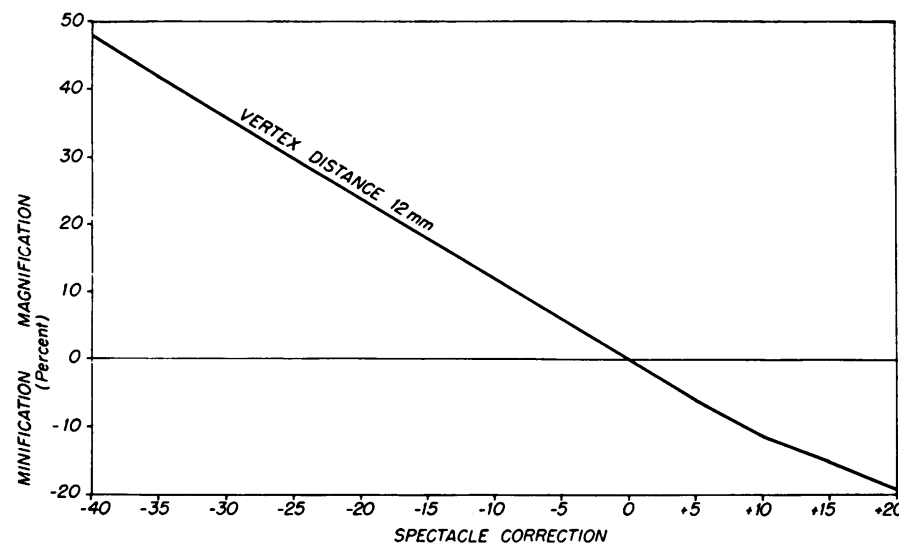

FIG. 3 Magnification produced by contact lenses in comparison with spectacle lenses

to a $-20 \mathrm{D}$ spectacle correction is 24 per cent. and for a - Io D spectacle lens 12 per cent. An additional 3 per cent. magnification is produced when the form and shape of lenses are considered. The data were calculated by J. L. Francis and published by Bennett ( 1963$)$.

The assumption that the magnification referred to the spectacle correction rather than to that on the corneal surface has left the belief that a magnification of 50 per cent. is produced by a $-20 \mathrm{D}$ spectacle lens as ordinarily understood. Boeder (1938) intended the magnification of 50 per cent. to refer to a contact lens correction, which is equivalent to a spectacle correction of $-26 \cdot 25 \mathrm{D}$ at a vertex distance of $\mathrm{I} 2 \mathrm{~mm}$.

The relation of the degree of effective magnification to the Snellen test chart is shown in Fig. 4 (overleaf).

Each line of letters on the charts using Sloan letters differs in steps of 26 per cent. A magnification of 26 per cent. enables the patient to read the next smaller line which is the degree of improvement made by fitting a patient wearing a spectacle correction of $-20 \mathrm{D}$ with a contact lens. This amounts to an improvement of vision from 6/18 to 6/15 (20/6o to $20 / 50) .^{*}$ Clinically, I have rarely ever made any greater improvement than this.

The diagnosis of seven patients in the Table (overleaf) was pathological myopia, in six congenital myopia, and in seven myopia secondary to the primary pathology such as retrolental fibroplasia. I differentiated congenital from pathological myopia when the patient manifested nystagmus. The significant data as far as this presentation is concerned are the visual acuities with spectacles and contact lenses. The visual improvement was frequently less than 26 per cent. even in very strong corrections. This Table shows that the clinical visual improvement was less than that calculated in most cases. For testing vision the Sloan letters were usually used. It is the best chart, because it includes $6 / 48$ (20/16o), 6/37.5 (20/125) and two lines for 6/30 (20/10o) and 6/24 (20/80). These letters are about as equal in legibility as can be obtained.

The corresponding spectacle and contact lens corrections agree reasonably well. However, the first case was either overcorrected with spectacles or wore the spectacle correction at a vertex distance of $19 \mathrm{~mm}$. Minus $25 \mathrm{D}$ contact lenses were prescribed first, and these were improved to $-2 \mathrm{I} \mathrm{D}$ by refracting over the first pair.

Of the eighteen patients who were followed, seven were judged to be failures and eleven to be successes. The criteria for success was whether the patient continued to wear the contact lenses. The principal cause of failure was inability to read with the contact lenses; this was the case in five patients. Highly myopic patients with subnormal vision

\footnotetext{
* The recorded measurements of acuity are based on measurements in feet, and for the convenience of readers using the metric system the equivalent at 6 metres is also given. No attempt has been made to equate to the Snellen distance types.
} 


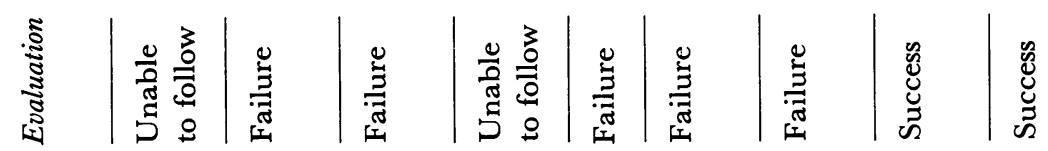

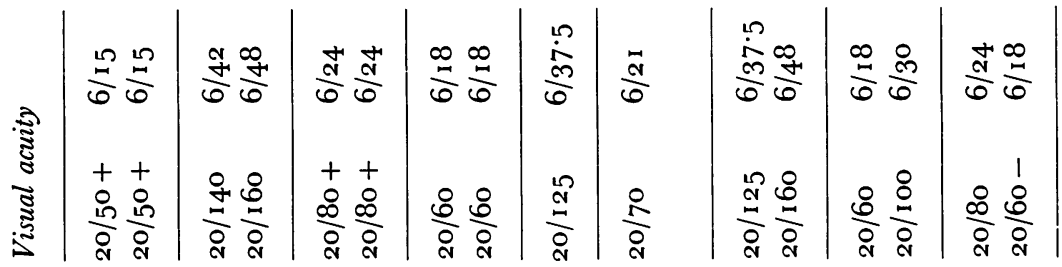

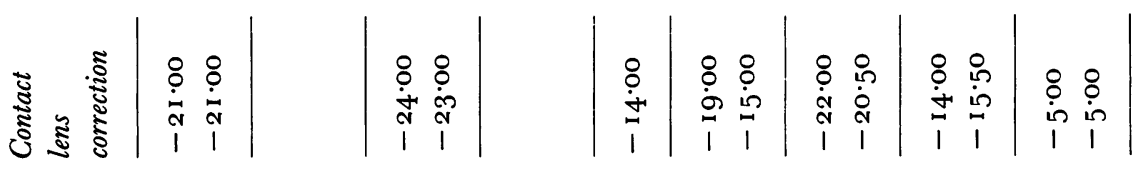

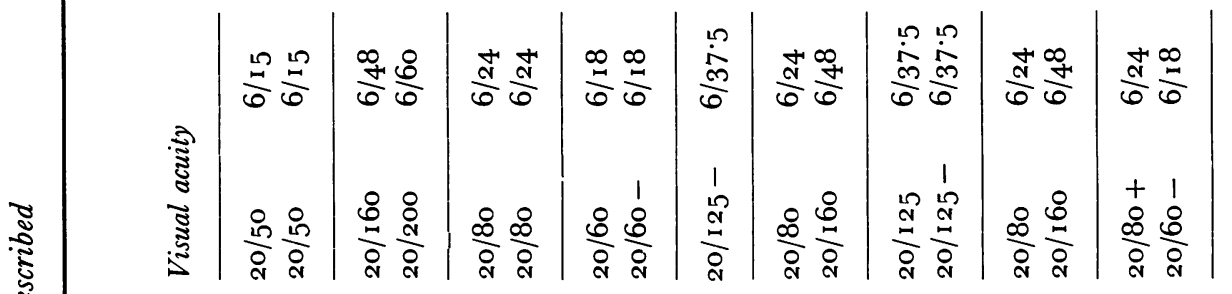

ह

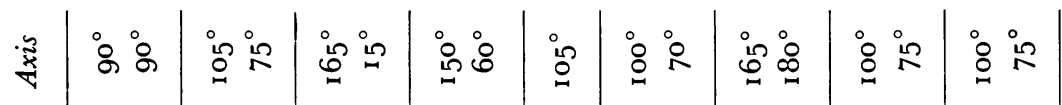

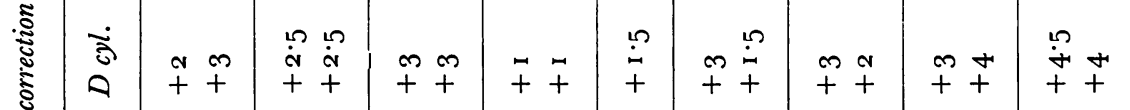

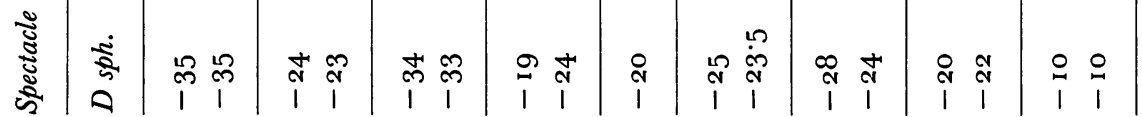

$\frac{\sqrt[3]{2}}{\sqrt[3]{2}}$

के

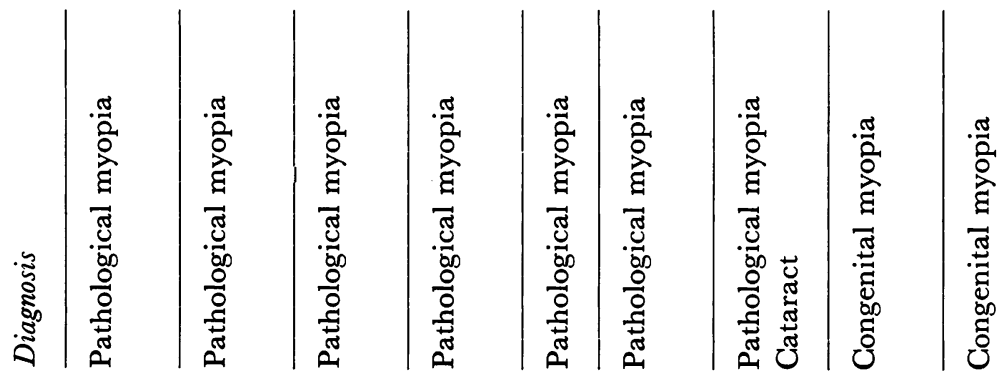

8

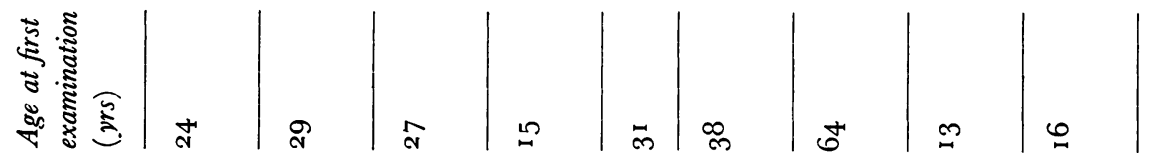

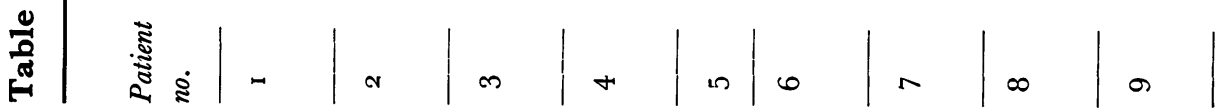

ฐ

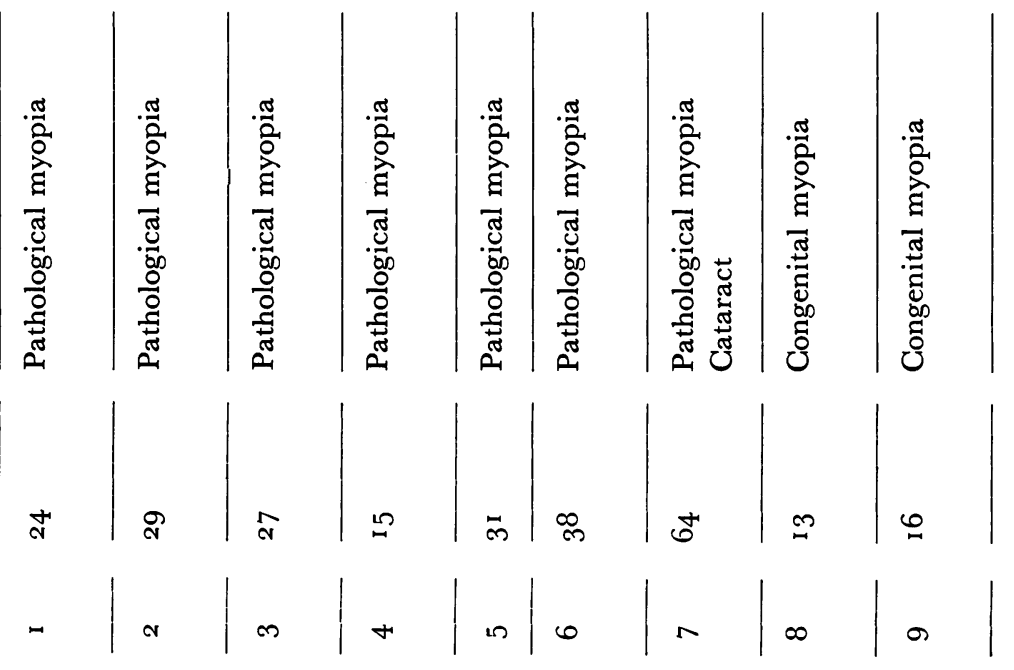




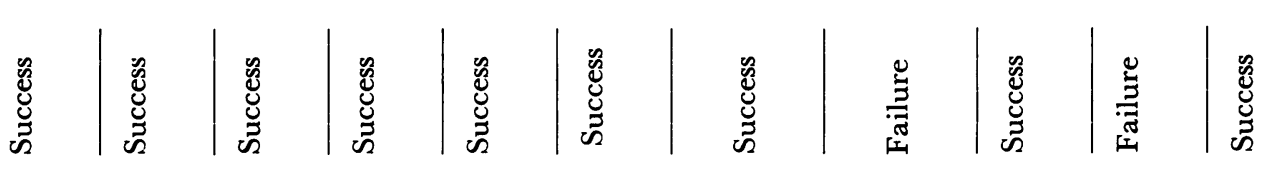

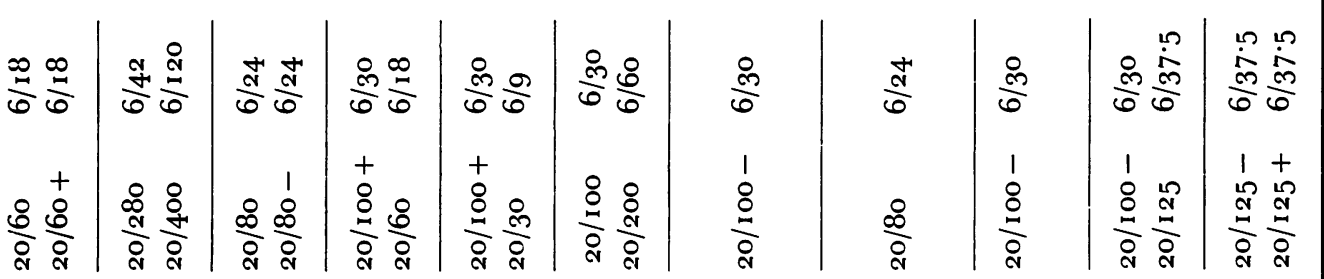

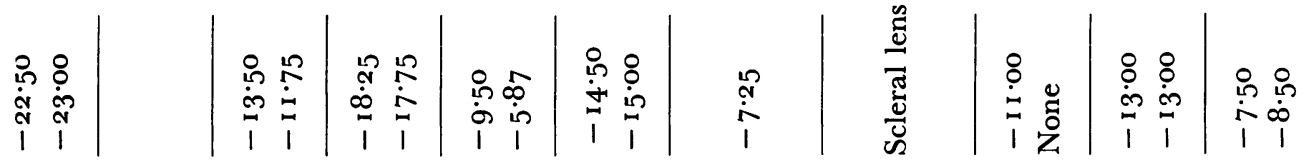

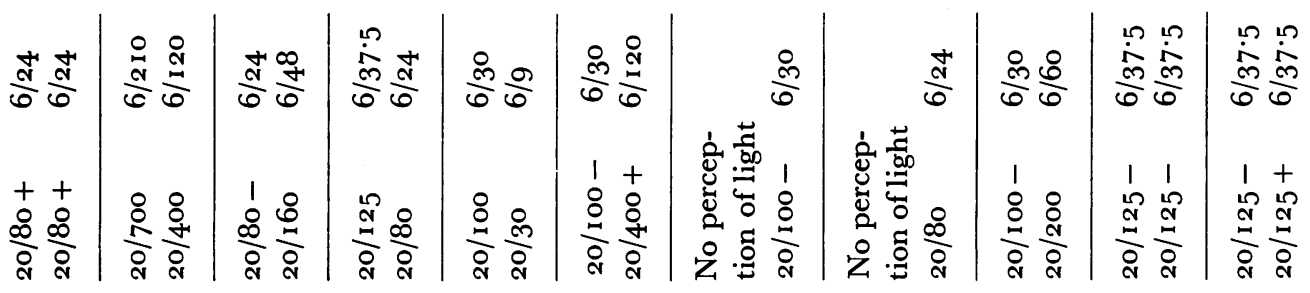

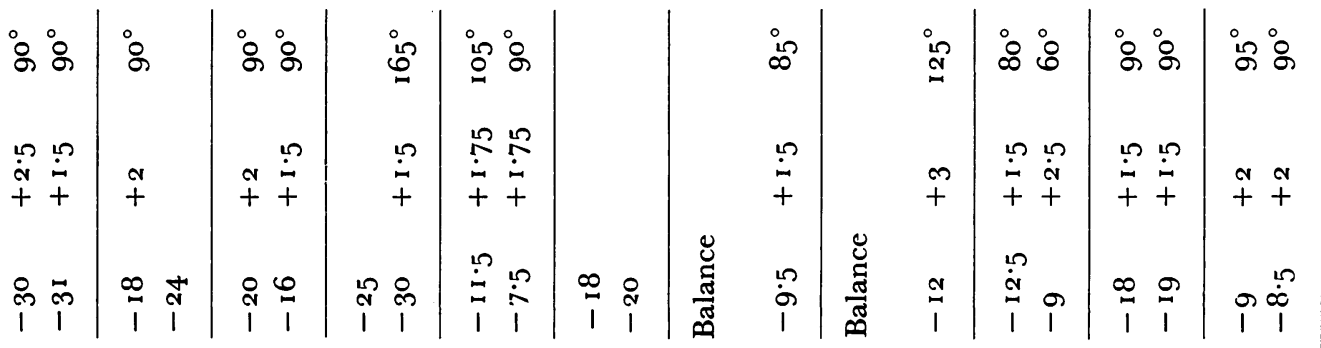

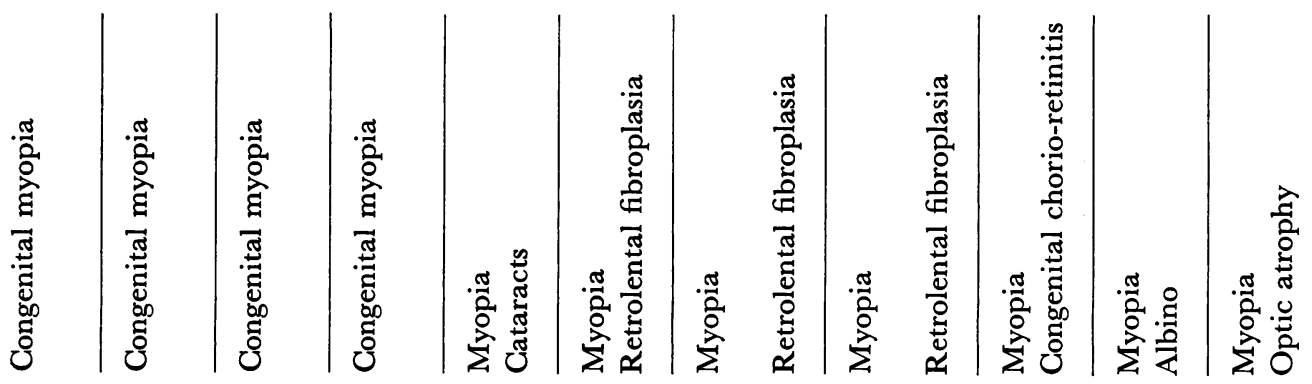

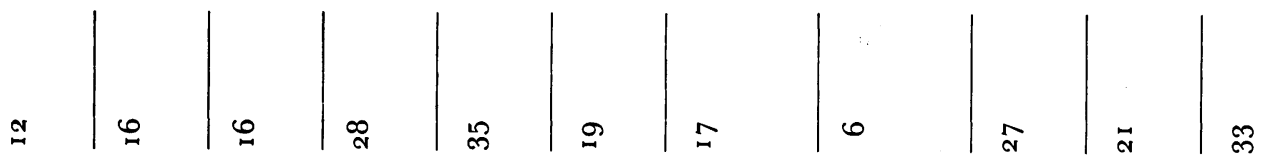

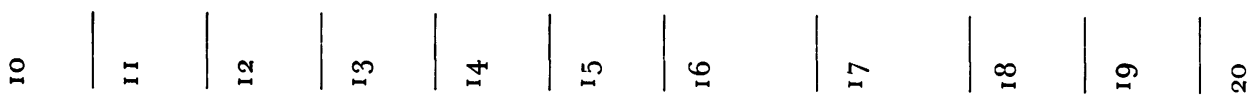



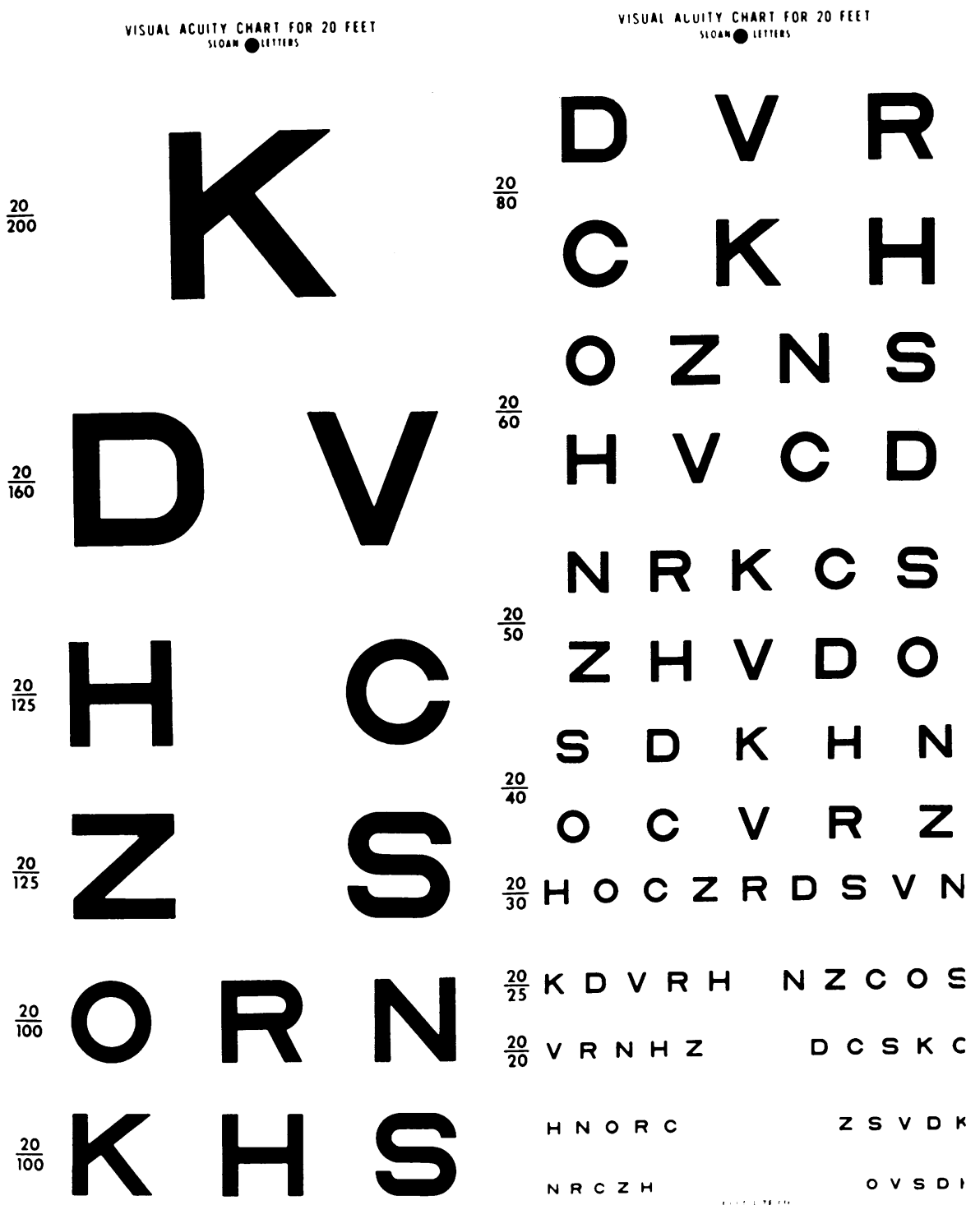

$\frac{20}{60}$
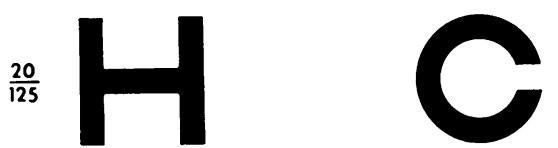

$\frac{20}{125}$

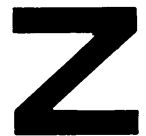

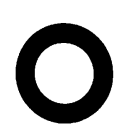
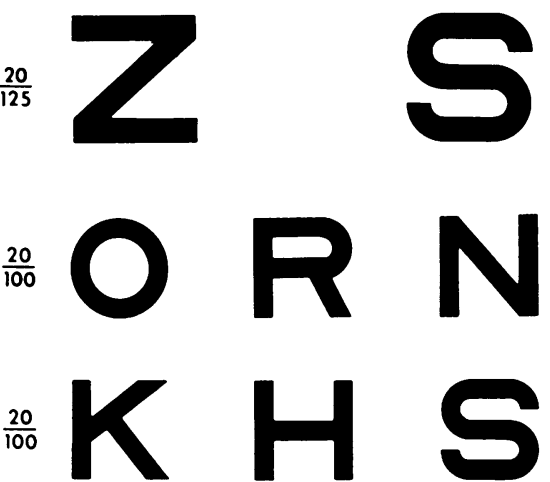

FIG. 4 Snellen test chart with Sloan letters

are fortunate because they have a built-in magnifier by just taking their glasses off to read at a distance of their far point or a little closer.

For distance, the contact lenses were better tolerated, and only two patients abandoned their use: one because of glare on lights and the other because his vision was not improved. The strong minus contact lenses proved to be as comfortable as weaker contact lenses. The higher the myopia the greater is the cosmetic advantage of contact lenses. The subjective improvement of more natural or better peripheral vision was not impressive. My experience leads me to conclude that the principal advantage of contact lenses in myopic patients with subnormal vision is essentially cosmetic. 


\section{Summary and conclusions}

The magnification of contact lenses in comparison to spectacle lenses is only half the amount which is the accepted belief. The magnification is 27 per cent. rather than $5^{\circ}$ per cent. for a contact lens in comparison to a $-20 \mathrm{D}$ spectacle correction fitted at a vertex distance of $12 \mathrm{~mm}$. This is a magnification equivalent to improving vision from 6/15 $(20 / 50)$ to $6 / 12(20 / 40)$ on the Snellen chart.

I have never rehabilitated a patient with subnormal vision associated with high myopia by prescribing contact lenses, nor have I ever seen one rehabilitated by anyone else.

The theory of lens correction of ametropia and magnification is presented. A graph illustrates the percentage of magnification by contact lenses in comparison to spectacle lenses.

The data of twenty cases of high myopia fitted with contact lenses are summarized. The clinically determined visual acuities with contact lenses in comparison to spectacle lenses are less than those calculated in many cases. The calculated and clinically determined magnification convinces me that contact lenses do not make a significant improvement in vision. The advantage of contact lenses for high myopia is essentially cosmetic.

Of the eighteen cases evaluated, eleven were judged to be successful and seven to be failures. Five of the failures were attributed to inadequate near vision, because the patient could no longer read at his uncorrected near point, which is easily done by lifting up his spectacles.

I wish to acknowledge the expert contributions of Dr. Donald Fonda.

\section{References}

BenNett, A. G. (1963) “Optics of Contact Lenses", pp. 6o, 61, 66. Association of Dispensing Opticians, London BOEDER, P. (1938) Arch. Ophthal. (Chicago), 19, 54 